\title{
Ramsey interferometry with a two-level Tonks-Girardeau gas
}

\author{
S. V. Mousavi* \\ Departamento de Química-Física, Universidad del País Vasco, Apdo. 644, 48080 Bilbao, Spain and \\ Department of Physics, Sharif University of Technology, P. O. Box 11365-9161, Tehran, Iran \\ A. del Campot I. Lizuain 7 and J. G. Muga \\ Departamento de Química-Física, Universidad del País Vasco, Apdo. 644, 48080 Bilbao, Spain
}

\begin{abstract}
We propose a generalization of the Tonks-Girardeau model that describes a coherent gas of cold two-level Bosons which interact with two external fields in a Ramsey interferometer. They also interact among themselves by contact collisions with interchange of momentum and internal state. We study the corresponding Ramsey fringes and the quantum projection noise which, essentially unaffected by the interactions, remains that for ideal Bosons. The dual system of this gas, an ideal gas of two-level Fermions coupled by the interaction with the separated fields, produces the same fringes and noise fluctuations. The cases of time-separated and spatially-separated fields are studied. For spatially separated fields the fringes may be broadened slightly by increasing the number of particles, but only for large particle numbers far from present experiments with TonksGirardeau gases. The uncertainty in the determination of the atomic transition frequency diminishes, essentially with the inverse root of the particle number.

PACS numbers: 03.75.-b, 03.75.Dg, 05.30.Jp
\end{abstract}

\section{INTRODUCTION}

A basic feature of the observed interference fringes in a standard Ramsey experiment is that their width is determined by the inverse of the time taken by the atoms to cross the intermediate drift region. For precision measurement purposes, as in atomic clocks, this motivates the use of very slow (ultracold) atoms and therefore the development of laser cooling techniques has changed the entire prospects of frequency standards [1]. Experimentally, atomic velocities of the order of $1 \mathrm{~cm} / \mathrm{s}$ and smaller can be achieved, and space-based clocks are in development to eliminate gravitational effects in the motion of such slow particles [2]. Laser cooled atoms are also interesting in metrology and interferometry because of the possibility to achieve narrow velocity distributions and avoid averaging effects. In addition, fundamentally new effects may arise by using coherent few-body or manybody states as input in the form of condensates or otherwise: for example, there exist proposals to beat the limitations imposed by quantum projection noise using entanglement [3, 4].

In spite of the above, the motto "the slower the better" in the context of atomic clocks has actually a limited domain beyond which quantum motional phenomena may affect strongly and eventually deform totally the usual Ramsey pattern. If the slow atom moves initially along the $x$-axis and the fields are oriented perpendicularly along the $y$-axis, there are two origins of modification

\footnotetext{
*Electronic address: s.v'moosavi@mehr.sharif.edu

${ }^{\dagger}$ Electronic address: adolfo.delcampo@ehu.es

‡Electronic address: ion.lizuain@ehu.es

§Electronic address: jg.muga@ehu.es
}

of the standard Ramsey result [5]. First, the absorption of a photon leads to a transverse momentum transfer on the atom, such that the excited state separates in space from the ground state. This is negligible for microwaves but not for optical transitions. The effect can be understood classically by means of energy conservation and momentum conservation in $y$-direction. It has been studied in detail by Bordé and coworkers [ 6 ] and multi-beam setups have been implemented to correct for this separation in order to observe quantum interference [7, 8, 9]. Second, the field acts as a barrier for the longitudinal motion of the atom, and quantum reflection and tunneling may occur. Thus, momentum in $x$-direction is not conserved as a consequence of the $x$-dependence of the fields. For microwave fields and the corresponding Rabi frequencies these quantized motion effects are tiny for present atomic velocities but may become important for deeply ultracold particles. Moreover, in view of a proclaimed near-future accuracy of frequency standards of $10^{-18}[10]$, even those tiny effects have to be studied beyond the limits of the standard theoretical description of the Ramsey pattern. Reflection effects have been considered for ultracold atoms passing through one [1] or two [12] resonant micromaser cavities, leading to the concept of "mazer physics". The nonresonant single-mazer case has been investigated by Bastin and Martin [13]. Recently, we extended the study to two separated classical fields and gave an exact quantum result of the Ramsey fringes as a function of the detuning including quantum tunneling and reflection by means of two-channel recurrence relations [14].

Apart from quantum motion effects affecting ensembles of independent particles, other effects are due to the importance of quantum statistics and interactions. The use of a Bose-Einstein condensate for an atomic clock immediately comes to mind, but the improvements associ- 
ated with low velocities and narrow velocity distribution may be compensated by negative effects, such as collisional shifts and instabilities leading to the separation of the gas cloud [15, 16].

A natural candidate for further exploration is the Tonks-Girardeau (TG) regime of impenetrable, tightly confined Bosons subjected to hard-core "contact" interactions [17, 18], since some of its properties are in a sense opposite to those of the condensate. In particular, the TG requirement of contact interactions, implies strong similarities between the Bosonic system and a "dual" system of freely moving Fermions, with all local correlation functions of both being actually equal. Other important feature of the TG gas is its one dimensional (1D) character. Olshanii showed [19, 20, 21] that when a Bosonic vapor is confined in a de Broglie wave guide with transverse trapping so tight and temperature so low that the transverse vibrational excitation quantum $\hbar \omega_{\perp}$ is larger than available longitudinal zero point and thermal energies, the effective dynamics becomes one dimensional, and accurately described by a 1D Hamiltonian with delta-function interactions $g_{1 D} \delta\left(x_{j}-x_{\ell}\right)$, where $x_{j}$ and $x_{\ell}$ are $1 \mathrm{D}$ longitudinal position variables. This is the Lieb-Liniger (LL) model, exactly solved in 1963 by a Bethe ansatz method 22]. The coupling constant $g_{1 D}$ can be tuned using a Feschbach resonance, allowing to reach the Tonks-Girardeau regime of impenetrable Bosons, which corresponds to the $g_{1 D} \rightarrow \infty$ limit of the LL model. It has been realized experimentally 23, 24], and was solved exactly in 1960 [17, 18] by the so called Fermi-Bose mapping to the ideal Fermi gas.

For metrology and interferometry applications the tight $1 \mathrm{D}$ confinement along a waveguide is a simplifying feature since no transversal motional branches have to be considered with the possible bonus of an increased signal. (The spatial separation into several branches may also be desirable, as in Sagnac interferometry, but it could be implemented with waveguides too.) Nevertheless, the confinement is by itself problematic for frequency standard applications, since it is carried out by means of magnetic or optical interactions which will in principle perturb the internal state levels of the atom. Several schemes have been proposed to mitigate this problem and compensate the shifts due to magnetic [15] or optical interactions $[25,26]{ }^{1}$

The possible applications in interferometry are a strong motivation for current research in TG gases. Interference effects have been examined so far in a few publications in which internal states have not played any role 27, 28]. Indeed, a TG model including internal states and an external interaction coupling them has not been discussed, although optically guided systems with free spin subjected to potentials for singlet and triplet interactions have been

\footnotetext{
${ }^{1}$ We shall assume hereafter that such compensation is implemented.
}

studied by means of effective LL models [29, 30]. Note also that a model applicable to a two-level LL gas coupled by an on-resonance laser has been solved by nested Bethe ansatz 31].

In this paper, we investigate the implications in Ramsey interferometry of a model in the spirit of the original (structureless) TG gas but with internal structure. The interactions defined allow us to achieve essential solvability of the dynamical problem in the Ramsey two-field excitation setup by simple quadrature: the collisions are characterized by internal state and momentum exchange, which reduce to the usual impenetrable constraint for collisions in the same internal channel.

We shall consider different configurations for the two fields, both in space and time domains. They are conceptually different and the mathematical treatment is different too. For reasonable parameters, however, the results turn out to be very similar.

\section{TWO-LEVEL TONKS-GIRARDEAU GAS WITH EXCHANGE, CONTACT INTERACTIONS}

We shall propose here a generalization of the TonksGirardeau gas for two-level impenetrable atoms. First we shall need to review or introduce some notation and basic concepts. In one dimension the state of a single two-level atom may be written as

$$
\Phi_{n}\left(x_{1}\right)=\sum_{b=g, e} \phi_{n}^{(b)}\left(x_{1}\right)|b\rangle
$$

where $n=1,2,3 \ldots$ is a label to distinguish different wave functions and $b$ is a generic index for the internal bound state which may be $g$ (ground), or $e$ (excited). Oneparticle states may be combined to form two-particle ones with the form

$$
\Phi_{n n^{\prime}}\left(x_{1}, x_{2}\right)=\sum_{b, b^{\prime}} \phi_{n}^{(b)}\left(x_{1}\right) \phi_{n^{\prime}}^{\left(b^{\prime}\right)}\left(x_{2}\right)\left|b b^{\prime}\right\rangle,
$$

and similarly for more particles. The convention in $\left|b b^{\prime}\right\rangle$ is that $b$ is for particle 1 and $b^{\prime}$ for particle 2 . This will in some equations be indicated even more explicitly adding a particle subscript to the internal state label, $b_{1}, b_{2}$, etc.

Consider now the usual Pauli operators acting on oneparticle internal state vectors,

$$
\begin{aligned}
\sigma_{X} & =|g\rangle\langle e|+| e\rangle\langle g|, \\
\sigma_{Y} & =i(|g\rangle\langle e|-| e\rangle\langle g|), \\
\sigma_{Z} & =|e\rangle\langle e|-| g\rangle\langle g|,
\end{aligned}
$$

and the corresponding 3-component operator $\hat{\mathbf{S}}_{j}=\boldsymbol{\sigma}_{j} / 2$ for particle $j$ analogous to the spin- $1 / 2$ angular momentum operator. If $\mathbf{S}=\mathbf{S}_{1}+\mathbf{S}_{2}, \mathbf{S}^{2}$ has eigenvalues $S(S+1)$ with $S=0$ and $S=1$ corresponding to singlet and triplet subspaces as it is well known. 
Assume now the following Hamiltonian

$$
\hat{H}_{\text {coll }}=-\frac{\hbar^{2}}{2 m} \sum_{j=1}^{2} \partial_{x_{j}}^{2}+v_{s}\left(x_{12}\right) \hat{P}_{12}^{s}+v_{t}\left(x_{12}\right) \hat{P}_{12}^{t} .
$$

Here $x_{12}=x_{1}-x_{2}$, and $\hat{P}_{12}^{s}=\frac{1}{4}-\hat{\mathbf{S}}_{1} \cdot \hat{\mathbf{S}}_{2}$ and $\hat{P}_{12}^{t}=$ $\frac{3}{4}+\hat{\mathbf{S}}_{1} \cdot \hat{\mathbf{S}}_{2}$ are the projectors onto the subspaces of singlet and triplet functions.

The internal Hilbert space can be written as $\mathcal{H}_{s} \oplus \mathcal{H}_{t}$, where $\mathcal{H}_{s}$ is spanned by $(|e g\rangle-|g e\rangle) / \sqrt{2}$ and $\mathcal{H}_{t}$ by $\{|g g\rangle,|e e\rangle,(|e g\rangle+|g e\rangle) / \sqrt{2}\}$. Suppose that the reflection amplitude for relative motion in such representation takes the values $+1,-1$ in singlet and triplet subspaces respectively. The particles are impenetrable and these values correspond to a hard wall potential $v_{t}$, whereas $v_{s}$ is a hard-core repulsive potential with an additional well of width $l$ and depth $V$, so that the reflection amplitude becomes $R=+1$ in the limit in which the well is made infinitely narrow and the well infinitely deep, keeping $\left(2 m V / \hbar^{2}\right)^{1 / 2} l=\pi / 2[29,32,33,34]$. Translated into the bare basis this implies that in all collisions between atoms in $g$ or $e$ and well defined momenta, they interchange their momenta (the relative momentum changes sign), as well as their internal state, with the outgoing wave function picking up a minus sign because of the hard-core reflection. For $x_{1}<x_{2}$ and equal internal states such collision is represented by

$$
e^{i k x_{1}} e^{i k^{\prime} x_{2}}|b b\rangle-\left[e^{i k^{\prime} x_{2}} e^{i k x_{1}}\right]|b b\rangle,
$$

whereas for $b \neq b^{\prime}$,

$$
e^{i k x_{1}} e^{i k^{\prime} x_{2}}\left|b b^{\prime}\right\rangle-\left[e^{i k^{\prime} x_{2}} e^{i k x_{1}}\right]\left|b^{\prime} b\right\rangle .
$$

In the diagonal case of equal internal states the spatial part vanishes at contact, $x_{1}=x_{2}$, whereas in the nondiagonal case it does not, but note that in Eq. (6) only the "external region" is considered, disregarding the infinitely narrow well region.

Up to now we have made no reference to quantum statistics and the particles are formally distinguishable. Let us consider now a Fermionic state made of freely moving one-particle states with the form

$$
\begin{aligned}
\Psi_{F}\left(x_{1}, x_{2}\right) & =\frac{1}{\sqrt{2}} \operatorname{det}_{n, m=1}^{2} \Phi_{n}\left(x_{m}\right) \\
& =\frac{1}{\sqrt{2}} \sum_{b_{1}, b_{2}=g, e}\left|\begin{array}{ll}
\phi_{1}^{\left(b_{1}\right)}\left(x_{1}\right) & \phi_{1}^{\left(b_{2}\right)}\left(x_{2}\right) \\
\phi_{2}^{\left(b_{1}\right)}\left(x_{1}\right) & \phi_{2}^{\left(b_{2}\right)}\left(x_{2}\right)
\end{array}\right|\left|b_{1} b_{2}\right\rangle,
\end{aligned}
$$

with the state sign changing by switching particles 1 and 2 and the internal states.

More explicitly, the different terms can be rearranged as

$$
\begin{aligned}
& 2^{1 / 2} \Psi_{F}\left(x_{1}, x_{2}\right)= \\
+ & {\left[\phi_{1}^{(g)}\left(x_{1}\right) \phi_{2}^{(g)}\left(x_{2}\right)-\phi_{2}^{(g)}\left(x_{1}\right) \phi_{1}^{(g)}\left(x_{1}\right)\right]|g g\rangle } \\
+ & {\left[\phi_{1}^{(e)}\left(x_{1}\right) \phi_{2}^{(e)}\left(x_{2}\right)-\phi_{2}^{(e)}\left(x_{1}\right) \phi_{1}^{(e)}\left(x_{2}\right)\right]|e e\rangle } \\
+ & {\left[\phi_{1}^{(g)}\left(x_{1}\right) \phi_{2}^{(e)}\left(x_{2}\right)\right]|g e\rangle-\left[\phi_{2}^{(e)}\left(x_{1}\right) \phi_{1}^{(g)}\left(x_{2}\right)\right]|e g\rangle } \\
+ & {\left[\phi_{1}^{(e)}\left(x_{1}\right) \phi_{2}^{(g)}\left(x_{2}\right)\right]|e g\rangle-\left[\phi_{2}^{(g)}\left(x_{1}\right) \phi_{1}^{(e)}\left(x_{2}\right)\right]|g e\rangle .(7) }
\end{aligned}
$$
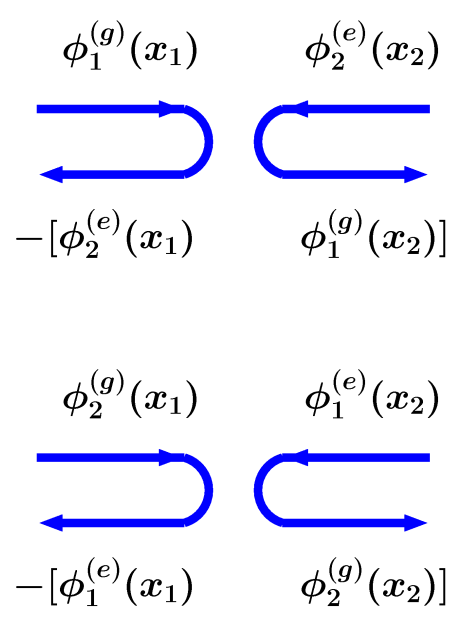

FIG. 1: Diagrammatic representation of the collisions for particles on different channels. Note that particles do not cross and interchange their momentum and internal state picking up an additional phase (minus sign).

This form makes evident that the Fermionic character of the state imposes for diagonal or non-diagonal terms the contact boundary conditions specified above, compare with Eqs. (556). An associated Bosonic system, totally symmetric under $\left(x_{i}, b_{i}\right) \leftrightarrow\left(x_{j}, b_{j}\right)$ permutations may be now obtained by means of the Bose-Fermi mapping, $\Psi\left(x_{1}, x_{2}\right)=\mathcal{A} \Psi_{F}\left(x_{1}, x_{2}\right)$, where the antisymmetric unit function is $\mathcal{A}=\operatorname{sgn}\left(x_{1}-x_{2}\right)$.

We have in summary constructed a Bosonic wave function for a system of two particles subjected to contact interactions with internal state and momentum interchange, using a dual system of two non-interacting Fermions and the antisymmetric unit function. The generalization to $N$-atoms is straightforward:

$$
\begin{aligned}
& \Psi_{F}\left(x_{1}, \ldots, x_{N}\right)=\frac{1}{\sqrt{N !}} \operatorname{det}_{n, m=1}^{N} \Phi_{n}\left(x_{m}\right) \\
= & \frac{1}{\sqrt{N !}} \sum_{b_{1}, \cdots, b_{N}=g, e}\left|\begin{array}{ccc}
\phi_{1}^{\left(b_{1}\right)}\left(x_{1}\right) & \cdots & \phi_{1}^{\left(b_{N}\right)}\left(x_{N}\right) \\
\vdots & \ddots & \vdots \\
\phi_{N}^{\left(b_{1}\right)}\left(x_{1}\right) & \cdots & \phi_{N}^{\left(b_{N}\right)}\left(x_{N}\right)
\end{array}\right|\left|b_{1} \cdots b_{N}\right\rangle,
\end{aligned}
$$

and

$$
\Psi\left(x_{1}, \ldots, x_{N}\right)=\mathcal{A} \Psi_{F}\left(x_{1}, \ldots, x_{N}\right),
$$

where

$$
\mathcal{A}=\prod_{1 \leq j<k \leq N} \operatorname{sgn}\left(x_{k}-x_{j}\right),
$$

is the Bosonic solution of the time-dependent or stationary Schrödinger equation for the Hamiltonian

$$
\hat{H}_{\text {coll }}=-\frac{\hbar^{2}}{2 m} \sum_{j=1}^{N} \partial_{x_{j}}^{2}+\sum_{1 \leq j<\ell \leq N}\left[v_{s}\left(x_{j \ell}\right) \hat{P}_{j \ell}^{s}+v_{t}\left(x_{j \ell}\right) \hat{P}_{j \ell}^{t}\right],
$$


with the same contact interactions as before.

The density profile, normalized to $N$-particles, which gives the appearance of the cloud, is defined by

$$
\rho_{N}(x)=N \int\left\|\Psi\left(x_{1}, \cdots, x_{N}\right)\right\|^{2} \mathrm{x}_{2} \cdots \mathrm{x}_{N} .
$$

Provided that the one-particle states $\Phi_{n}$ are orthonormal, as they will always be hereafter, the density profile reads

$$
\rho_{N}(x)=\sum_{b=g, e} \sum_{n=1}^{N}\left|\phi_{n}^{(b)}(x)\right|^{2}=\sum_{b=g, e} \rho_{N}^{(b)}(x),
$$

where the density profile for each of the channels defined by the two internal levels is given by

$$
\rho_{N}^{(b)}(x)=\sum_{n=1}^{N}\left|\phi_{n}^{(b)}(x)\right|^{2}
$$

The simplicity achieved by our model parallels that of the usual (structureless) TG gas in the sense that an $N$-body wavefunction with interactions is obtained from freely-moving one-body states. Even more, this property is preserved by adding an interaction affecting the individual atoms only and coupling the internal levels. This is precisely the type of interaction that we find in the Ramsey interferometer.

\section{QUANTUM PROJECTION NOISE IN A TWO-LEVEL TONKS-GIRARDEAU GAS}

Itano and coworkers 36 studied the quantum projection noise for a Hartree product state of the form $\left|b_{1}, \ldots, b_{N}\right\rangle=\otimes_{i=1}^{N}\left|b_{i}\right\rangle$. This noise is, in other words, the fluctuation of the number of excited atoms for measurements made in the $N$-body system. In Ramsey interferometry, the error in the determination of the atomic frequency depends on the ratio between the (root of the) fluctuation in the number of excited atoms and the derivative of the signal (proportional to the number of excited atoms) with respect to detuning.

Here we shall obtain the noise associated with the state $\Psi\left(x_{1}, \cdots, x_{N}\right)$. We shall follow 36] and introduce the operator

$$
\hat{S}_{Z}=\sum_{i=1}^{N} \hat{S}_{i_{Z}}=\frac{1}{2} \sum_{i=1}^{N}\left(\left|e_{i}\right\rangle\left\langle e_{i}|-| g_{i}\right\rangle\left\langle g_{i}\right|\right),
$$

where it is assumed, as usual, that each term in the summation is multiplied by the identity operator for all the other atoms.

The quantum projection noise of a signal is proportional to the variance

$$
\left(\Delta S_{Z}\right)^{2}=\left\langle\hat{S}_{Z}^{2}\right\rangle-\left\langle\hat{S}_{Z}\right\rangle^{2},
$$

and expressions for both terms will now be worked out. First, notice that $S_{Z}$ commutes with $\mathcal{A}$ so that, using $\mathcal{A}^{2}$, we may compute the expectation values substituting $\Psi$ by $\Psi_{F}$, i.e., for the more easily tractable, dual Fermionic system. Since $\Psi_{F}$ is antisymmetric it follows that

$$
\left\langle\hat{S}_{Z}\right\rangle=N\left\langle\Psi\left|\hat{S}_{1_{Z}}\right| \Psi\right\rangle
$$

and

$$
\begin{aligned}
\left\langle\hat{S}_{Z}^{2}\right\rangle & =\sum_{i=1}^{N}\left\langle\hat{S}_{i_{Z}}^{2}\right\rangle+\sum_{i, j}\left\langle\hat{S}_{i \neq j} \hat{S}_{j_{Z}}\right\rangle \\
& =N\left\langle\hat{S}_{1_{Z}}^{2}\right\rangle+N(N-1)\left\langle\hat{S}_{1_{Z}} \hat{S}_{2_{Z}}\right\rangle .
\end{aligned}
$$

Eq. (16), takes the form

$$
\begin{aligned}
N\left\langle\Psi\left|\hat{S}_{1_{Z}}\right| \Psi\right\rangle & =N \int \prod_{i} \mathrm{~d} x_{i} \Psi^{*}\left(x_{1}, \ldots, x_{N}\right) \hat{S}_{1_{Z}} \Psi\left(x_{1}, \ldots, x_{N}\right) \\
& =\sum_{n=1}^{N}\left\langle\Phi_{n}\left|\hat{S}_{1_{Z}}\right| \Phi_{n}\right\rangle=\frac{1}{2} \sum_{n=1}^{N} \alpha_{n}
\end{aligned}
$$

where $\alpha_{n}=p_{n}^{(e)}-p_{n}^{(g)}$ is the probability difference for the excited and ground state in state $n$.

In Eq. (17), note that $S_{1_{Z}}^{2}=\mathbf{1}_{N} / 4$ and therefore $\left\langle\hat{S}_{1_{Z}}^{2}\right\rangle=1 / 4$ for the normalized state $\Psi\left(x_{1}, \cdots, x_{N}\right)$. The cross term can be evaluated as

$$
\begin{aligned}
& N(N-1)\left\langle\hat{S}_{1_{z}} \hat{S}_{2_{z}}\right\rangle \\
= & \sum_{n, m}\left(\left\langle\Phi_{n}\left|\hat{S}_{1_{Z}}\right| \Phi_{n}\right\rangle\left\langle\Phi_{m}\left|\hat{S}_{2_{Z}}\right| \Phi_{m}\right\rangle-\left\langle\Phi_{n} \Phi_{m}\left|\hat{S}_{1_{z}} \hat{S}_{2_{Z}}\right| \Phi_{m} \Phi_{n}\right\rangle\right) \\
= & \frac{1}{4} \sum_{n, m}\left(\alpha_{n} \alpha_{m}-\Delta_{n m}\right),
\end{aligned}
$$

where the $\Delta_{n m}$ terms are positive and defined as

$$
\begin{aligned}
\Delta_{n m} & =\left|\int \mathrm{d} x\left[\phi_{n}^{(e)}(x)\right]^{*} \phi_{m}^{(e)}(x)-\left[\phi_{n}^{(g)}(x)\right]^{*} \phi_{m}^{(g)}(x)\right|^{2} \\
& =\left|\left\langle\phi_{n}^{(e)} \mid \phi_{m}^{(e)}\right\rangle-\left\langle\phi_{n}^{(g)} \mid \phi_{m}^{(g)}\right\rangle\right|^{2}
\end{aligned}
$$

Combining these results, the variance simply reads

$$
\left(\Delta S_{Z}\right)^{2}=\frac{N}{4}-\frac{1}{4} \sum_{n, m} \Delta_{n m}
$$

If the dependence of single particle expectation value $\left\langle\Phi_{n}\left|\hat{S}_{1_{z}}\right| \Phi_{n}\right\rangle$ on $n$ can be neglected, so that $\alpha_{n} \simeq \alpha$ for all $n$,

$$
\begin{aligned}
\left(\Delta S_{Z}\right)^{2} & =\frac{N}{4}\left(1-\frac{1}{N} \sum_{n} \alpha_{n}^{2}\right)-\frac{1}{4} \sum_{n, m n \neq m} \Delta_{n m} \\
& \simeq \frac{N}{4}\left(1-\alpha^{2}\right)-\frac{1}{4} \sum_{n, m n \neq m} \Delta_{n m} \\
& <\left(\Delta S_{Z}\right)_{0}^{2}
\end{aligned}
$$

where we have identified a term $\left(\Delta S_{Z}\right)_{0}^{2} \equiv \frac{N}{4}\left(1-\alpha^{2}\right)$ corresponding to the quantum noise for the Hartree product state in [36], and a negative correction for the strongly interacting Bosonic TG gas. 


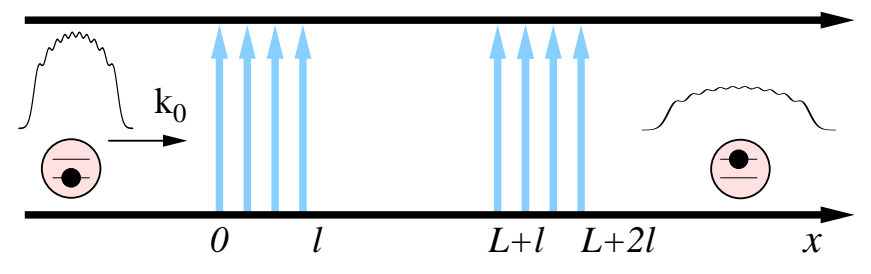

FIG. 2: Schematic setup for Ramsey interferometry of guided atoms in the spatial domain. The atoms are prepared in the ground state and the probability of excitation is measured after passing the two fields.

\section{THE RAMSEY INTERFEROMETER}

Ramsey interferometry with guided ultracold atoms has recently been discussed in [14]. Here we consider a system of $N$ two-level atoms in the Tonks-Girardeau regime, initially confined in their ground internal states in a harmonic trap of frequency $\omega$. All energy scales are supposed to be much smaller than the transverse excitation energy $\hbar \omega_{\perp}$, so that the radial degrees of freedom are frozen out and the system is effectively onedimensional. The cloud is prepared in the ground state, and released by switching off the trap at time $t=0$; a momentum kick $\hbar k_{0}$ is also applied, so that the cloud moves along the $x$ axis towards the two separated oscillating fields localized between 0 and $l$ and between $l+L$ and $2 l+L$ (Fig. 22). The initial state is prepared far from the first field. We thus have to take into account the spatial width (root of the variance) of the highest state, $\delta_{N}=[(N+1 / 2) \hbar /(m \omega)]^{1 / 2}$, and choose the central initial position of the harmonic trap $x_{0}<0$ so that $x_{0}<<\delta_{N}$.

In an oscillating-field-adapted interaction picture and using the Lamb-Dicke (see the next section), dipole and rotating-wave approximations the Hamiltonian is, for each of the particles,

$$
H=\frac{\widehat{p}^{2}}{2 m}-\hbar \Delta|e\rangle\langle e|+\frac{\hbar}{2} \Omega(\widehat{x})(|g\rangle\langle e|+| e\rangle\langle g|),
$$

where the first term counts for the kinetic energy of the atom, $\Delta=\omega_{L}-\omega_{12}$ is the detuning between the oscillating field frequency and atomic transition frequency, and $\Omega(x)$ is the position-dependent Rabi frequency. For the explicit $x$ dependence we assume mesa functions, $\Omega(x)=\Omega$ for $x \in[0, l]$ and $x \in[l+L, 2 l+L]$ and zero elsewhere. In addition, we have to include the interparticle interactions but this is done implicitly by means of the wave function (8) and its boundary conditions at contact.

The Ramsey pattern is defined by the dependence on the detuning of the probability of excited atoms after the interaction with the two field regions. From Eq. (13) it follows that $P_{e}^{(N)}=\sum_{n=1}^{N} P_{e}^{(n)}$, which is a remarkably simple result for an $N$-body system with external and interparticle interactions. Once a particle incident from the left and prepared in the state $e^{i k_{0}\left(x-x_{0}\right)} \phi_{n}\left(x-x_{0}\right)|g\rangle$

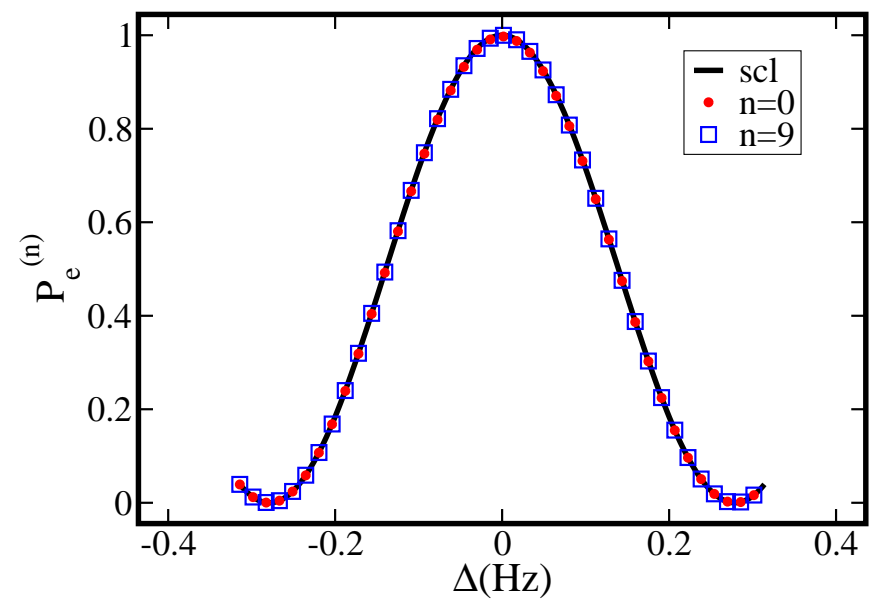

FIG. 3: Central fringe for Ramsey interferometry in the spatial domain. The agreement is shown for the $n=0,9$ singleparticle wavepackets and the semiclassical result, for ${ }^{133} \mathrm{Cs}$ atoms, with $\hbar k_{0} / m=1 \mathrm{~cm} / \mathrm{s}, l=1 \mathrm{~cm}, L=10 \mathrm{~cm}$, and $t=15 \mathrm{~s}$.

at $t=0$ has passed completely through both fields, the probability amplitude for it to be in the excited state is

$$
\phi_{n}^{(e)}(x, t)=\frac{1}{\sqrt{2 \pi}} \int \mathrm{k} e^{i q x-i k^{2} \hbar t /(2 m)} T_{g e}(k) \tilde{\phi}_{n}(k),
$$

where $\tilde{\phi}_{n}(k)$ is the wavenumber representation of the kicked $n$-th harmonic eigenstate,

$$
\begin{aligned}
\tilde{\phi}_{n}(k) & =\frac{(-i)^{n}}{\sqrt{2^{n} n !}}\left(\frac{2 \delta_{0}^{2}}{\pi}\right)^{1 / 4} \\
& \times e^{-\delta_{0}^{2}\left(k-k_{0}\right)^{2}} e^{-i k x_{0}} H_{n}\left[\sqrt{2} \delta_{0}\left(k-k_{0}\right)\right],
\end{aligned}
$$

the momentum in the excited state is $q=\sqrt{k^{2}+2 m \Delta / \hbar}$, the spatial width of the $n=0$ state is $\delta_{0}=[\hbar /(2 m \omega)]^{1 / 2}$, $H_{n}$ the Hermite polynomials, and $T_{g e}$ is the "doublebarrier" transmission amplitude for the excited state corresponding to atoms incident in the ground state (the excited state probability for monochromatic incidence in the ground state is $\frac{q}{k}\left|T_{g e}\right|^{2}$ ). The full quantum treatment of $T_{g e}$ can be done by means of the two-channel recurrence relations connecting it with one-field transmission and reflection amplitudes 14].

Our numerical simulations are for $l=1 \mathrm{~cm}, L=10$ $\mathrm{cm}, N=10$, and $v_{0}=1 \mathrm{~cm} / \mathrm{s}$. Figure 3 shows that the variation of the excitation probability for different harmonic eigenstates is negligible in the scale shown, and in fact the curves for the central fringe are indistinguishable from the semiclassical result of Ramsey (which assumes classical motion for the center of mass, uncoupled from the internal levels),

$$
P_{12}(\Delta)=\frac{4 \Omega^{2}}{\Omega^{\prime 2}} \sin ^{2}\left(\frac{\Omega^{\prime} \tau}{2}\right)\left[\cos \left(\frac{\Omega^{\prime} \tau}{2}\right) \cos \left(\frac{\Delta T}{2}\right)\right.
$$




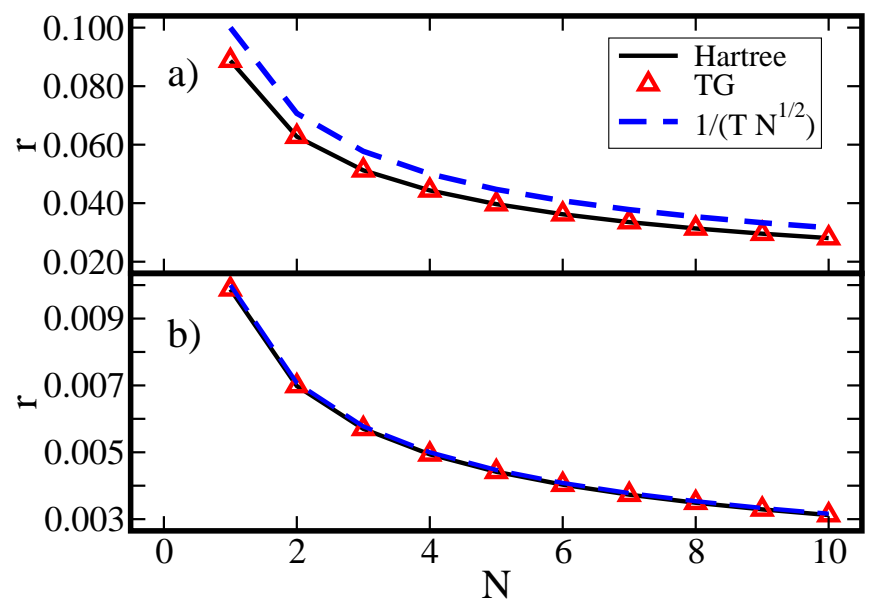

FIG. 4: Quantum projection noise ratio, Eq. (30), for the Ramsey interferometry in the spatial domain. The two-level Tonks-Girardeau gas ratio is essentially that of a Hartree product (uncorrelated atoms). The same parameters as in Fig. 3 are used, with a) $L=10 \mathrm{~cm}$, b) $L=100 \mathrm{~cm}$.

$$
\left.-\frac{\Delta}{\Omega^{\prime}} \sin \left(\frac{\Omega^{\prime} \tau}{2}\right) \sin \left(\frac{\Delta T}{2}\right)\right]^{2},
$$

where $\tau=l / v_{0}, T=L / v_{0}$, and $\Omega^{\prime}=\left(\Omega^{2}+\Delta^{2}\right)^{1 / 2}$.

There is in principle a broadening of the central fringe by increasing $n$ due to the momentum broadening of vibrationally excited states. This effect may be expected however to be quite small for the few-body states of our calculations, $N=10$, which is in fact of the order of current experiments with TG gases $(N \approx 15,50$ in [23, 24]) The width (root of the variance) of the velocity distribution around the central velocity $v_{0}=\hbar k_{0} / m$ for the $n$-th state is

$$
\Delta_{v}=\left[\left(n+\frac{1}{2}\right) \frac{\omega \hbar}{m}\right]^{1 / 2}=\sqrt{2 n+1} \frac{\hbar}{2 m \delta_{0}},
$$

where we have used the spatial width of the $n=0$ state, $\delta_{0}=[\hbar /(2 m \omega)]^{1 / 2}$. This will not affect significantly the width of the central fringe (proportional to the inverse of the crossing time $T$ ) as long as $\Delta_{v} / v_{0}<<1$. For $v_{0}=1$ $\mathrm{cm} / \mathrm{s}, \delta_{0}=20 \mu \mathrm{m}$, the mass of ${ }^{133} \mathrm{Cs}$, and $N=10$, this ratio is $\sim 5 \times 10^{-3}$. $N$ should be $\sim 4 \times 10^{5}$ to get a ratio of order one, but this means four orders of magnitude more particles than in the existing experiments.

The error to estimate the atomic frequency from the Ramsey pattern depends on the ratio

$$
r=\frac{\Delta S_{Z}}{\left|\partial\left\langle S_{Z}\right\rangle / \partial \Delta\right|}
$$

which we calculate at half height of the central interference peak. We compute $\Delta S_{Z}$ with Eq.(22). Since, according to the previous discussion, the excitation probabilities are essentially independent of $n, \mathrm{Eq} .(23)$ is an excellent approximation. Moreover, the correction to $\left(\Delta S_{Z}\right)_{0}$ due the particle correlations is negligible, with a relative error $\left[\left(\Delta S_{Z}\right)_{0}-\Delta S_{Z}\right] / \Delta S_{Z} \sim 10^{-10}$ in our calculations. Since, in addition, the derivative in Eq. (30) is very well approximated by the semiclassical result, the ratio $r$ essentially coincides with that for freely moving, uncorrelated particles [36] and, for $L>>l$ it gives $1 /(T \sqrt{N})$ for all $\Delta$, see Fig. 4,

\section{RAMSEY INTERFEROMETRY IN THE TIME DOMAIN FOR GUIDED ATOMS}

An alternative to the previous set-up is the separation of the fields in time rather than space but, at variance with the usual procedure, keeping the gas confined transversally at all times as required for the $1 D$ regime of the TG gas, Fig. 5] Because of the tight confinement the transverse vibrational excitation is negligible so that the Ramsey pattern is given by the standard expression irrespective of the value of $n$. The whole TG gas therefore produces the usual Ramsey pattern (28) as we shall see in more detail.

A two level atom in a cigar shape trap with characteristic frequencies $\omega_{x}, \omega_{y}$ and $\omega_{z}\left(\omega_{x} \ll \omega_{y} \sim \omega_{z}\right)$ interacting with a (classical) laser field directed in the perpendicular $y$ direction is described (in a laser adapted interaction picture) by the Hamiltonian

$$
\begin{aligned}
H= & \sum_{i=x, y, z} \hbar \omega_{i}\left(a_{i}^{\dagger} a_{i}+\frac{1}{2}\right)-\hbar \Delta|e\rangle\langle e| \\
& +\frac{\hbar \Omega}{2}\left[e^{i \eta_{y}\left(a_{y}+a_{y}^{\dagger}\right)} \sigma_{+}+H . c\right]
\end{aligned}
$$

where $\sigma_{+}=|e\rangle\langle g|$. The Rabi frequency $\Omega$ is here a constant, independent of $x$, and $a_{i}^{\dagger}\left(a_{i}\right)$ are the creation (annihilation) operators of the vibrational modes in the direction of the subscript. The parameter $\eta_{y}=k_{L} y_{0}$ is known as the Lamb-Dicke (LD) parameter, with $y_{0}=$ $\sqrt{\hbar / 2 m \omega_{y}}$ being the extension of the atomic ground state in $y$-direction. The vibrational modes in the longitudinal $x$-direction are not coupled by the field if the $x$ dependence of the field is negligible in the scale $\delta_{N}$ of the cloud. Also, motion in the $z$-direction remains uncoupled.

\section{A. Lamb-Dicke regime}

A particular interesting limit when dealing with trapped atoms interacting with laser fields is the so called Lamb-Dicke regime. In this regime, the extension of the atom's wave function in the direction of the field is much smaller than the laser wavelength, i.e., $\eta_{y} \ll 1$. If the LD regime is assumed, it is natural to approximate the exponentials in the coupling term of the Hamiltonian (31) 


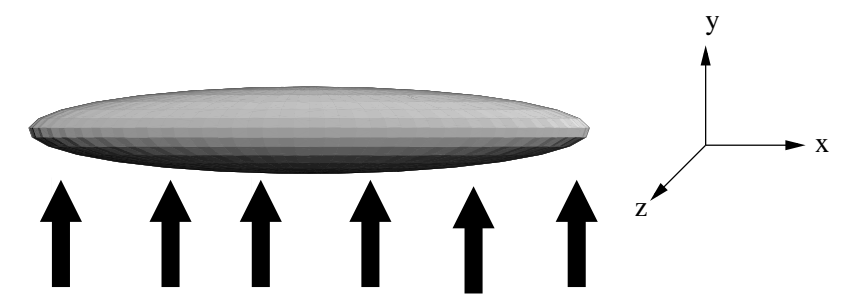

FIG. 5: Schematic setup for Ramsey interferometry in time domain. The TG gas is confined in a cigar-shaped trap and illuminated by a laser in $y$-direction.

by $e^{ \pm i \eta_{y}\left(a_{y}+a_{y}^{\dagger}\right)} \approx 1$, giving an approximate Hamiltonian

$$
\begin{aligned}
H_{L D}= & \sum_{i=x, y, z} \hbar \omega_{i}\left(a_{i}^{\dagger} a_{i}+\frac{1}{2}\right)-\hbar \Delta|e\rangle\langle e| \\
& +\frac{\hbar \Omega}{2}\left(\sigma_{+}+\sigma_{-}\right)
\end{aligned}
$$

which does not couple the vibrational modes in the transversal $y$-direction. In this regime, the vibrational levels are well separated and the fields cannot induce transitions between them (the recoil frequency is much smaller than the trapping frequency). Within this approximation the number operators $n_{i}=a_{i}^{\dagger} a_{i}$ are some constant of motion for $i=x, y, z$ and thus the dynamics of the system is independent of the vibrational modes, reproducing the usual Ramsey fringe pattern (28) when time separated pulses are applied.

\section{B. TG regime}

A tight transversal confinement is needed in order to reach the TG regime, which is achieved when the dimensionless parameter $\gamma=m g / \hbar^{2} n \gg 1$. Here $n \approx N / \delta_{N}$ is the linear density of the gas and $g$ the $1 \mathrm{D}$ interaction strength, which is given by $g=2 \hbar^{2} a / m y_{0}^{2}$, with $a$ being the s-wave scattering length [37]. We may then write the criterion for being in the TG regime as $2 a / n y_{0}^{2} \gg 1$ or

$$
\gamma=\frac{2 a \delta_{N}}{N y_{0}^{2}} \gg 1
$$

If ${ }^{133} \mathrm{Cs}$ atoms in a trap with frequency $\omega_{y} \approx 2 \pi \times$ $1 \mathrm{MHz}$ are considered, the transversal confinement turns out to be $y_{0} \approx 6 \mathrm{~nm}$. For $\delta_{0} \approx 20 \mu \mathrm{m}$, a scattering length of $a \approx 100 a_{0}, a_{0}$ being the Bohr radius, and $N=10$, $\gamma \approx 2 \times 10^{3}$, well in the TG regime.

If the hyperfine transition of the ${ }^{133} \mathrm{Cs}$ atom at $9.192 \mathrm{GHz}$ is driven, a LD parameter of $\eta_{y}=k_{L} y_{0} \approx 10^{-6}$ is obtained for the transversal confinement of $y_{0} \approx 6 \mathrm{~nm}$ previously estimated, which is well inside the $\mathrm{LD}$ regime. The TG condition then imposes the LD condition for microwave transitions. For optical transitions, LD parameters of $0.05-0.1$ are obtained, that can also be considered to lay into the LD regime.

\section{SUMMARY AND DISCUSSION}

A model of $N$ Bosons in 1D with contact interactions that interchange the momentum and internal state of the 2-level atoms subjected to two oscillating fields has been worked out.

A word is in order about the interactions between different channels. In the singlet component, the space wavefunction is antisymmetric, so that s-wave scattering is forbidden, and the interactions are governed to leading order by a $3 \mathrm{D}$ p-wave scattering amplitude. In close analogy with spin-polarized Fermions, such interactions can be enhanced by a p-wave Feschbach resonance, with an associated 1D odd-wave confinement-induced one-dimensional Feschbach resonance (CIR) which allows to engineer $v_{s}[29,32,33,35]$.

For realistic parameters in the ultracold regime the system behaves similarly for spatial or temporal separation of the fields, and according to the semiclassical Ramsey pattern for independent, freely moving particles. Moreover, the quantum projection noise reminds close to that of an ensemble of independent atoms.

For the two-level Tonks-Girardeau gas, the interactions do not worsen the quality of the Ramsey pattern but have the additional advantage of dramatically reducing the three-body correlation function [38, 39] and therefore enhancing the stability of the gas with respect to the ideal case. We expect strongly interacting gases to play a remarkable role in interferometry with ultracold atoms in waveguides.

\section{Acknowledgements}

We acknowledge discussions with M. Girardeau. This work has been supported by Ministerio de Educación y Ciencia (FIS2006-10268-C03-01) and UPV-EHU (00039.310-15968/2004). S. V. M. acknowledges a research visitor Ph. D. student fellowship by the Ministry of Science, Research and Technology of Iran. A. C. acknowledges financial support by the Basque Government (BFI04.479). 
lomon et al., C. R. Acad. Sci. Paris Série IV 2, 1313 (2001).

[3] W. J. Wineland et al., Phys. Rev. A 46, R6797 (1992).

[4] S. F. Huelga et al., Phys. Rev. Lett. 79, 3865 (1997).

[5] N. F. Ramsey, Phys. Rev. 78, 695 (1950); N. F. Ramsey, Molecular beams (Clarendon Press, Oxford, 1956), Chapter V.4.

[6] C. J. Bordé, C. R. Acad. Sci. Paris Série IV 2, 509 (2001); P. Wolf et al., in Proc. 6th Symp. on Frequency Standards and Metrology, 2002, edited by P. Gill (World Scientific, 2002), p. 593; C. J. Bordé, Metrologia 39, 435 (2002).

[7] C. J. Bordé, C. Salomon, S. Avrillier, A. van Lerberghe, C. Bréant, D. Bassi, and G. Scoles, Phys. Rev. A 30, 1836 (1984).

[8] M. Kasevich and S. Chu, Phys. Rev. Lett. 67, 181 (1991); Appl. Phys. B 54, 321 (1992).

[9] A. Morinaga, Phys. Rev. A 45, 8019 (1992).

[10] S. Schiller et al., arXiv:gr-qc/0608081

[11] B. G. Englert, J. Schwinger, A. O. Barut, and M. O. Scully, Europhys. Lett. 14, 25 (1991); M. O. Scully, G. M. Meyer, and H. Walther, Phys. Rev. Lett. 76, 4144 (1996).

[12] G. S. Agarwal and R. Arun, Phys. Rev. Lett. 84, 5098 (2000); R. Arun and G. S. Agarwal, Phys. Rev. A 64, 065802 (2001).

[13] T. Bastin and J. Martin, Phys. Rev. A 67, 053804 (2003).

[14] D. Seidel and J. G. Muga, Eur. Phys. J. D 41, 71 (2007).

[15] D. M. Harber, H. J. Lewandowski, J. M. Mc Guirk, and E. A. Cornell, Phys. Rev. A 66, 053616 (2002).

[16] D. Kadio and Y. B. Band, Phys. Rev. A 74, 053609 (2006).

[17] M. D. Girardeau, J. Math. Phys. 1, 516 (1960).

[18] M. D. Girardeau, Phys. Rev. 139, B500 (1965), Secs. 2, 3 , and 6 .

[19] M. Olshanii, Phys. Rev. Lett. 81, 938 (1998).
[20] T. Bergeman, M. Moore M, and M. Olshanii, Phys. Rev. Lett. 91, 163201 (2003).

[21] D. S. Petrov, G. V. Shlyapnikov, and J. T. M. Walraven, Phys. Rev. Lett. 85, 3745 (2000).

[22] E. H. Lieb and W. Liniger, Phys. Rev. 130, 1605 (1963).

[23] B. Paredes et al., Nature 429, 277 (2004).

[24] T. Kinoshita, T. R. Wenger, and D. S. Weiss, Science 305, 1125 (2004).

[25] A. Kaplan, M. F. Andersen, and N. Davidson, Phys. Rev. A 66, 045401 (2002).

[26] H. Hafner et al., Phys. Rev. Lett. 90, 143602 (2003).

[27] M. Girardeau and E. M. Wright, Phys. Rev. Lett. 84, 5239 (2000).

[28] M. D. Girardeau, K. K. Das, and E. M. Wright, Phys. Rev. A 66, 023604 (2002).

[29] M. D. Girardeau, H. Nguyen, and M. Olshanii, Optics Communications 243, 3 (2004).

[30] M. D. Girardeau, Phys. Rev. Lett. 97, 210401 (2006).

[31] C. N. Yang, Phys. Rev. Lett. 19, 1312 (1917); M. Gaudin, Phys. Lett. A 24, 55 (1917); X. W. Guan et al., cond-mat/0702191.

[32] M. D. Girardeau and M. Olshanii, cond-mat/0309396.

[33] M. D. Girardeau and M. Olshanii, Phys. Rev. A 70, 023608 (2004).

[34] T. Cheon and T. Shigehara, Phys. Lett. A 243, 111 (1998); Phys. Rev. Lett. 82, 2536 (1999).

[35] B. E. Granger and D. Blume, Phys. Rev. Lett. 92, 133202 (2004).

[36] W. M. Itano et al., Phys. Rev. A 47, 3554 (1993).

[37] J. Reichel, J. H. Thwissen J. Phys. IV France 116, 265 (2004).

[38] D. M. Gangardt and G. V. Shlyapnikov, Phys. Rev. Lett. 90, 010401 (2003).

[39] A. del Campo, J. G. Muga, and M. D. Girardeau, arXiv:0705.0937 